\title{
Forecasting Of Type-2 Fuzzy Electric Power System Based On Phase Space Reconstruction Model
}

\author{
Juan Zhao and Lihui Jiang \\ Department of Mathematics and Physics, Hefei University, Hefei Anhui, China, \\ 230601
}

\begin{abstract}
Type-2 fuzzy logic to make up for the lack of a type of fuzzy logic in dealing with uncertainty, object contains uncertainty is strong; the application of type-2 fuzzy logic advantage is more obvious. In this paper, type-2s of fuzzy logic for power load time series forecasting, good results were obtained. According to the power load has strong randomness it is difficult to accurately forecast problem, the introduction of the type-2 of fuzzy logic in order to reduce the prediction error. According to the power load is difficult to predict accurately the problem, this paper introduces the interval type-2 fuzzy logic method to reduce the prediction error, presents an interval type-2 fuzzy logic model for the time series of one hour of power load forecasting, and adopted the first modeling process model structure, and then use back propagation algorithm to adjust the model parameters are determined by simulation. At the same time, based on the backpropagation algorithm of a fuzzy model with interval type-2 fuzzy model, singular value decomposition iterative blending algorithm of interval type-2 fuzzy model, the predicted result was the test performance benchmark. The simulation results show that the prediction accuracy of the forecasting model, based on the established with high, can better track the actual load curve, a better performance than the other three models.
\end{abstract}

Keywords: Type-2 fuzzy sets, forecasting, reconstruction, similarity measure

\section{Introduction}

The type- 2 of fuzzy logic based on the type-2 fuzzy system identification is an effective method for nonlinear system identification, type-2 fuzzy logic by a unified type fuzzy logic and expansion, can use linguistic experience of human experts, has a unique advantage in dealing with uncertainty, is suitable for complex non-linear systems with stochastic disturbance is serious a type of fuzzy logic [1]. Compared with that of type-2 fuzzy logic to type-2 fuzzy sets as a basis, the parameters of membership functions of adjustable increased, and by extending the synthesis to complete the fuzzy reasoning process, thus obtaining a better ability to deal with uncertainty $[2,3]$.

Fuzzy measure is to promote play its role is an important tool of the fuzzy logic better, a type of measure of fuzziness in fuzzy system identification has unique properties, use its can simultaneously reduce redundant fuzzy sets and redundant fuzzy rules; and in the type- 2 of fuzzy system identification, similar research is still few [4]. This paper the emphasis is put on the type- 2 of fuzzy measure and its application of fuzzy system identification in type-2, the adverse effect to eliminate the redundant fuzzy sets and redundant fuzzy rules brings; reduced model constructed for power load time series prediction, in order to improve the prediction accuracy [5].

Electric power load forecasting is an important work in industry, accurate electricity load forecast is reasonable power system planning, construction, production, scheduling, and an important basis for maintenance, you can ensure 
network security, economy of operation, power enterprises to improve the economic and social benefits [6]. However, the power load fluctuation is a non-stationary random process, affected by many natural and social factors, various factors is uncertain, so it's very difficult to accurately predict. as the electricity market deepening reform, uncertainties inherent in the power system makes decisionmaking is facing a certain degree of risk, and must be considered in the decisionmaking power demand uncertainty. in this context, finding an effective tool dealing with uncertainty methods to improve the accuracy of power load forecasting has very important significance. the traditional forecasting methods due to the existence of many problems and difficult to obtain the desired results, so people turning their attention to a number of modern methods based on artificial intelligence theory on which type-2 fuzzy logic has strong processing capacity of uncertainty for time series prediction, for the power load forecasting provides a new way of thinking [7, $8,9]$.

To Interval type- 2 fuzzy similarity paper, wood for the interval type-2 fuzzy system identification, we propose a counter-propagating a singular value decomposition of a hybrid similarity iterative algorithm. On the basis of the use of back-propagation algorithm to adjust system parameters, through the interval type- 2 fuzzy similarity to identify redundant fuzzy rule base of fuzzy sets, the merger means to eliminate the deleted, thus reducing the fuzzy rules library If the number of redundancy is strong, but also can reduce the redundancy of fuzzy rules by means of merger; the number of fuzzy sets needed to improve the interpretable fuzzy rules on the basis of risk and the use of the singular value decomposition method is preferable blur rules. In short, the algorithm can effectively eliminate the adverse effects of the redundancy of fuzzy sets and fuzzy rules bring redundancy and reduce the computational complexity of the fuzzy reasoning process and improve the approximation accuracy of the system.

\section{Related Work}

\subsection{Type-2 Fuzzy Sets}

Defined on a continuous domain on $\mathrm{X}$ type-2 fuzzy set $\mathrm{A}$ can be expressed as:

$$
\bar{A}=\int_{x \in X} \int_{u \in J_{x}} \mu_{\bar{A}}(x, u) /(x, u)
$$

In the formula: $\mu_{\bar{A}}(x, u) \in[0,1]$ is the membership function; $u \in J_{x}$ is the main membership values; Jx Union called the uncertainty of the trace; Type-2 fuzzy sets are given based on fuzzy degree of membership of the element, its membership function is three-dimensional, a type of fuzzy sets more than one dimension, which means that multiple one-dimensional description and processing the uncertainty of the degrees of freedom, thus enhanced the collection capability of dealing with uncertainties, but also increase the complexity of the set [10].

For type-2 fuzzy set theory any domain A point $\mathrm{x}=\mathrm{x}$, Two practice fuzzy membership function ( $x$ ) with the plane $x=x$, the intersection. Called secondary membership function (Secondary MF), formula (2)

$$
\mu_{\bar{A}}\left(x=x^{\prime}, u\right)=\mu_{\bar{A}}\left(x=x^{\prime}\right)=\int_{u \in J_{x^{\prime}}} f_{x^{\prime}}(u) / u
$$

In it: $u \in J_{x^{\prime}} \subseteq[0,1], 0 \leq f_{x^{\prime}}(u) \leq 1$

A can be expressed as a function of all times and set membership, such as the formula (3)

$$
\bar{A}=\int_{x \in X} \mu_{\bar{A}}(x) / x=\int_{x \in X}\left[\int_{u \in J_{x}} f_{x}(u) / u\right] / x
$$




\subsection{Triangle Type-2 Fuzzy Logic System}

A definition in the $\mathrm{X}$ domain of the type-2 fuzzy set is as follows:

$$
\begin{aligned}
\tilde{A} & =\int_{x \in X}\left[\int_{u \in J_{x}} \mu_{\tilde{A}}(x)\right] / x \\
& =\int_{x \in X}\left[\int_{u \in J_{x}} f_{x}(\mathrm{u}) / u\right] / x
\end{aligned}
$$

Where: $u$ is the element of the $X$ main membership values, $F x(U)$ is a membership value, the range of $\mathrm{Jx} u$ and the uncertainty of a trace (FOU), FOU, respectively, lower limit of the corresponding membership function.

Figure 1 is a triangle type- 2 fuzzy set: the domain $x=[0,5]$, when $x=3$, the main membership is time interval $[0.5,1]$, membership function is a triangle function curve ACA correspondences.

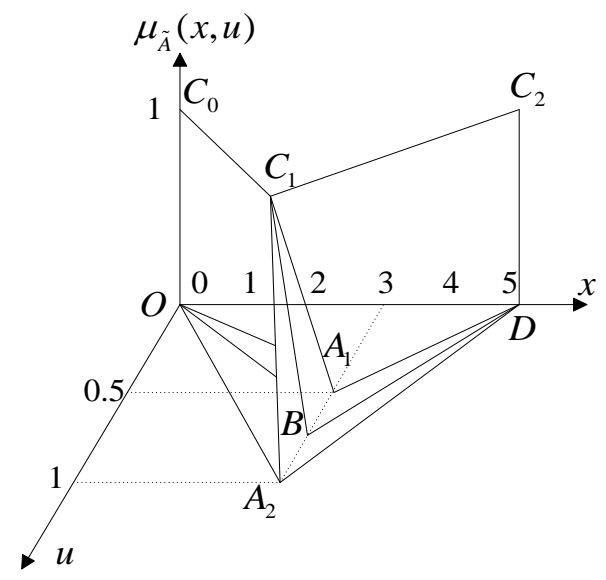

\section{Figure 1. The Triangle Type-2 Fuzzy Sets}

The time interval of type- 2 fuzzy set membership values are 1 , as shown in the following formula:

$$
\tilde{A}=\int_{x \in X}\left[\int_{u \in J_{x}} 1 / u\right] / x
$$

The fuzzy set of interval type-2 is a simplified version of the type-2 fuzzy sets, avoids the choice of membership functions, and the pay, compensation, and calculation are greatly simplified, so it has practical value.

\subsection{The Shortcoming of the Current Type-2 Fuzzy Sets Theory}

The design of the excellent performance of type-2 of fuzzy system identification is necessary, but the research about this aspect is not many, there are many technical / bottleneck, wherein the redundant problem of fuzzy rule base to be solved, including two aspects of fuzzy redundancy and the redundant fuzzy rules set, reducing the fuzzy rules can explain sex, cause / dimension disaster, an increase of unnecessary computation, and affect the accuracy of the system. How to type-2 fuzzy system rule base slim is a research hotspot, present a few fuzzy rule base reduction method is only for the redundant fuzzy rules streamlined, and can not effectively eliminate the redundancy in the adverse effect of fuzzy set.

Type-2 fuzzy logic has strong ability to deal with the uncertainty of the design, the excellent performance of type- 2 of fuzzy system identification is necessary, but the research on this aspect has just started, there are many shoe problem to be solved, including redundant fuzzy rule base is one of the most common problems. 
Type-2 fuzzy logic consists of a type of fuzzy logic and expansion, so a lot of fuzzy system identification method can be extended to type-2s of fuzzy system identification, a type of measure of fuzziness in fuzzy principle of application in system identification can be extended to type- 2 fuzzy system identification. Type-2s of fuzzy logic and fuzzy logical although have a lot in common, but also has its own characteristics.

\section{The Type-2 of Fuzzy System Rule Base Simplification Algorithm}

\subsection{Interval Type-2 Fuzzy Similarity}

A real function $N: I V F S s \times I V F S s \rightarrow[0,1]$ satisfies the following conditions, called the fuzzy similarity interval type-2, when $\mathrm{N}$ satisfies the following axioms:

$$
\begin{aligned}
& (N 1) N(\tilde{A}, \tilde{B})=N(\tilde{B}, \tilde{A}) ; \\
& (N 2) N\left(D, D^{c}\right)=0, \forall D \in P(X) ; \\
& (N 3) N(\tilde{E}, \tilde{E})=\max _{\tilde{A} \tilde{B} \in I V F S S} N(\tilde{A}, \tilde{B}) ;
\end{aligned}
$$

Interval two main membership function of fuzzy set selection variance of uncertainty the Gauss function, membership function, as shown in the following formula. The model has 3 inputs, 1 output, that is, with the first 3 moments of the load values to predict values after a moment.

$$
\underline{\mu}_{X_{k}}\left(x_{k}\right)=\exp \left[-\frac{1}{2}\left(\frac{x_{k}-x_{k}^{*}}{\underline{\sigma}_{k}}\right)^{2}\right]
$$

Where: $\mathrm{x}{ }_{\mathrm{k}}$ is the exact input values, $\left[\underline{\sigma}_{k}, \bar{\sigma}_{k}\right]$ is variation range of variance; $\mathrm{K}$ $=1, \ldots \mathrm{p}$, is the input dimension, $\mathrm{p}=3$. Each of the front parts of the input space consists of three sets, so the rule base containing $\mathrm{M}$ complete $=3 * 3 * 3=27$ rule. Due to the adoption of Center - of - sets drop type, it can be set after the piece with (interval set) instead, as shown in eq.:

$$
\bar{\mu}_{F \xi}\left(x_{k}\right)=\left\{\begin{array}{lr}
\exp \left[-\frac{1}{2}\left(\frac{x_{k}-\underline{m}_{k}^{l}}{\sigma_{k}^{l}}\right)^{2}\right], & x \leq \underline{m}_{k}^{l}, \\
1, & \underline{m}_{k}^{l} \leq x \leq \underline{m}_{k}^{l}, \\
\exp \left[-\frac{1}{2}\left(\frac{x_{k}-\bar{m}_{k}^{l}}{\sigma_{k}^{l}}\right)^{2}\right], & x>\bar{m}_{k}^{l},
\end{array}\right.
$$

There are three definitions in the discrete domain of $X=\{x 1, X 2, x 3\}$ on the interval type-2 fuzzy sets A, B, C, as shown below:

$$
\begin{aligned}
& \mu_{\tilde{A}}\left(x_{1}\right)=\frac{1}{0.2}+\frac{1}{0.4}, \mu_{\tilde{A}}\left(x_{2}\right)=\frac{1}{0.4}+\frac{1}{0.5} \\
& \mu_{\tilde{B}}\left(x_{1}\right)=\frac{1}{0.1}+\frac{1}{0.3}, \mu_{\tilde{B}}\left(x_{2}\right)=\frac{1}{0.2}+\frac{1}{0.4} \\
& \mu_{\tilde{C}}\left(x_{1}\right)=\frac{1}{0.4}+\frac{1}{0.5}, \mu_{\tilde{C}}\left(x_{2}\right)=\frac{1}{0.4}+\frac{1}{0.6}
\end{aligned}
$$

After a similar calculation, get the fuzzy similarity to other, listed in Table 1: 
Table 1. The Fuzzy Similarity Listed

\begin{tabular}{cccccc}
\hline & $\tilde{A}_{1}$ & $\tilde{A}_{2}$ & $\tilde{A}_{3}$ & $\tilde{A}_{4}$ & $\tilde{A}_{5}$ \\
\hline$\tilde{A}$ & 1.0 & 0.0029 & 0.0138 & 0.3282 & 0.0001 \\
& 000 & & & & \\
$\tilde{A}_{2}$ & 0.0 & 1.0000 & 0.3973 & 0.0197 & 0.2069 \\
& 029 & & & & \\
$\tilde{A}$ & 0.0 & 0.3973 & 1.0000 & 0.0702 & 0.0697 \\
& 138 & & & & \\
$\tilde{A}_{4}$ & 0.3 & 0.0197 & 0.0702 & 1.0000 & 0.0015 \\
& 282 & & & & \\
$\tilde{A}$ & 0.0 & 0.2069 & 0.0697 & 0.0015 & 1.0000 \\
& 001 & & & & \\
\hline
\end{tabular}

\subsection{SVD-SM-BP Hybrid Iterative Algorithm}

For a collection of highly overlapping, can be used to identify the fuzzy similarity, combined to produce a public collection, and then use the public collection to replace these overlapping set.

$$
\begin{gathered}
\bar{m}_{k}^{l q}=\lambda_{1} \bar{m}_{k}^{l}+\left(1-\lambda_{1}\right) \bar{m}_{k}^{q} \\
\underline{m}_{k}^{l q}=\lambda_{2} \underline{m}_{k}^{l}+\left(1-\lambda_{2}\right) \underline{m}_{k}^{q} \\
\sigma_{k}^{l q}=\lambda_{3} \sigma_{k}^{l}+\left(1-\lambda_{3}\right) \sigma_{k}^{q}
\end{gathered}
$$

If there are two similar interval type- 2 fuzzy sets, $\left[\underline{m}_{k}^{l}, \bar{m}_{k}^{l}\right]=[59,63]$ respectively $\left[\underline{m}_{k}^{q}, \bar{m}_{k}^{q}\right]=[65,70]$, the mean variance respectively $\underline{m}_{k}^{l q}=\frac{\underline{m}_{k}^{l}}{2}+\frac{\underline{m}_{k}^{q}}{2}=62$, the replacement ensemble FOU mean is shown in Figure 2.

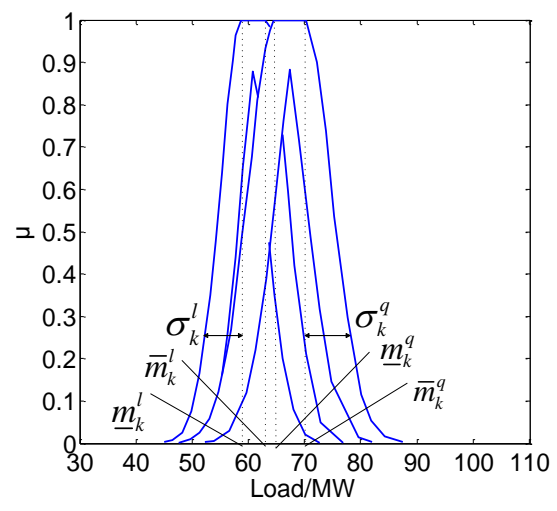

Figure 2. (a) FOUs of Interval Type-2 Fuzzy Sets $\tilde{F}_{k}^{l}$ and $\tilde{F}_{k}^{q}$ 


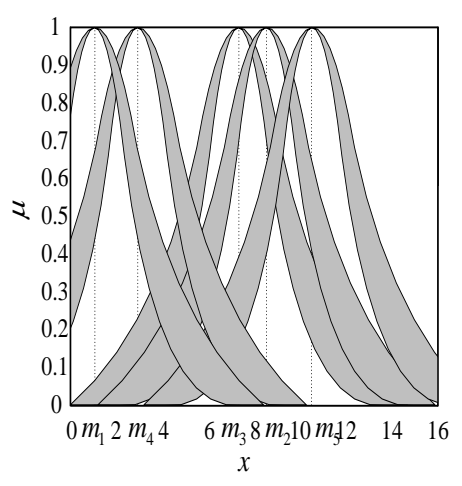

Figure 2. (b) FOU of Alternative Set $\tilde{F}_{k}^{l q}$

The calculation of the output of the fuzzy system: $Y(x)=a / b$. The system can be viewed as a three layer feed forward neural network, as shown in Figure 3

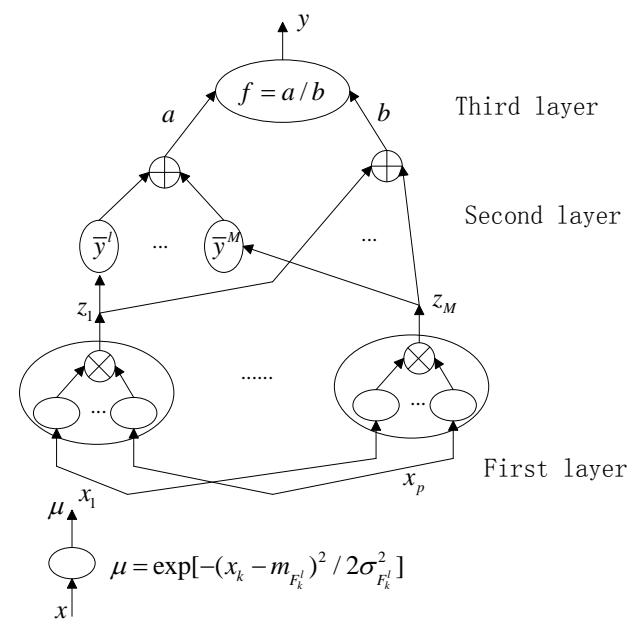

Figure 3. The System Viewed As A Three Layer Feed Forward Neural Network

The output interval of type-2 fuzzy systems from input and fuzzy before rule sets, under the right set of centroid of membership function and the consequent, the left endpoint decision. For each set of input, there are two decision system output of a fuzzy system for a range of type-2 fuzzy systems; respectively is the interval of type-2 fuzzy systems left right, extended fuzzy basis function. So, using the algorithm to adjust real interval type- 2 fuzzy system parameter is adjusting the two parameters of a fuzzy system.

\subsection{Type-2 Fuzzy Logic System}

Type-2 fuzzy logic system based on type-2 fuzzy sets generally includes the fuzzifier, rule base, the inference engine, drop type device and the defuzzification part five, as shown in Figure 4, a type of fuzzy logic system of more than one drop type links. 


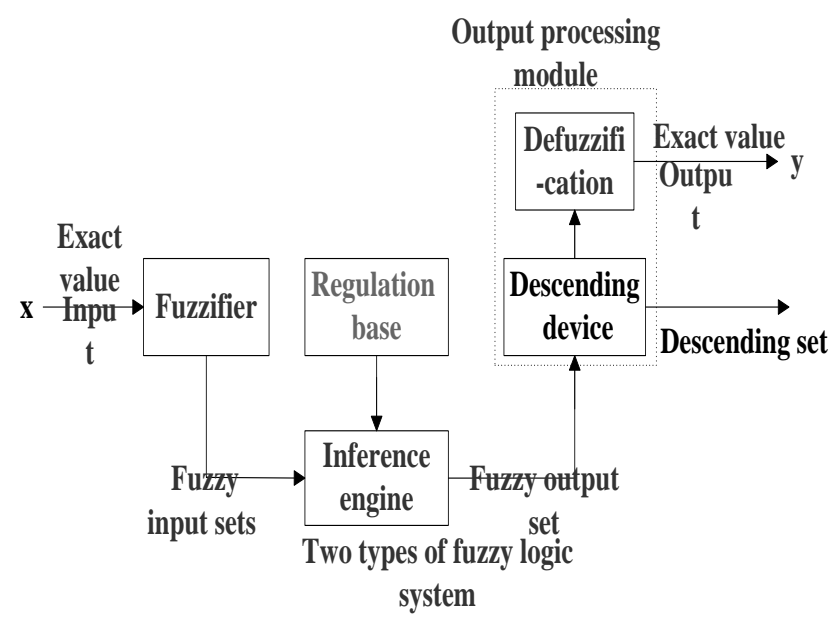

Figure 4. The Inference Engine, Drop Type Device and the Defuzzification

Compared with the first type fuzzy logic systems, type- 2 fuzzy logic system in construction and reasoning mode of the system and there is no significant change, but it is to type-2 fuzzy sets as a basis, the adjustable parameter increased, increasing the number of degrees of freedom can be adjusted, so as to obtain the better ability of handling uncertainty. Ordinary the type-2 of fuzzy logic system, realize the difficult and complicated calculation, application of interval type- 2 fuzzy logic system can reduce the amount of calculation, and simplifies the reasoning process.

\section{Experimental Results}

\subsection{The Simulation and Verification}

We can see from the process of modeling and simulation results, a model with 24 rules, the prediction error is $21.67 \%$. Model two based on model SVD adverse effects on the use of redundant fuzzy rules brought elimination method, 20 rules only, make the prediction error down to $18.95 \%$. The simulation curve shown in Figure 5 is consistent with the above analysis: the two models can well follow the trend and direction of the actual wind speed curve, but the predicted curve model two is better than one prediction curve fitting performance model.

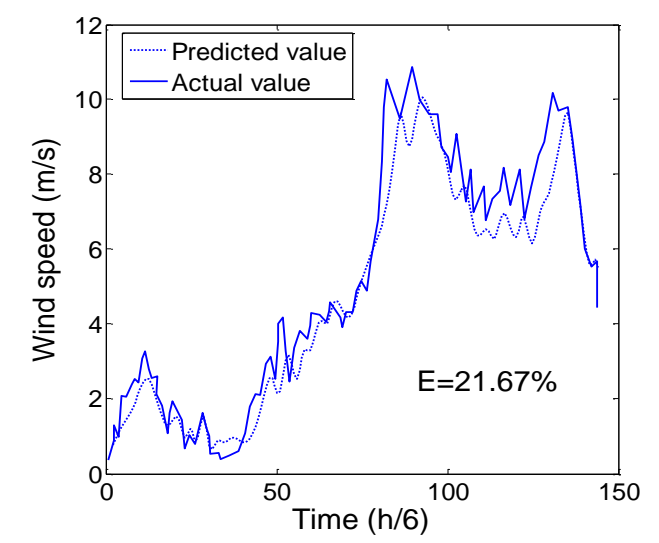

Figure 5. The Prediction Results of the Two Models 
This section introduces the prediction error interval type-2 fuzzy logic to reduce the wind speed, the establishment of an interval BP algorithm two non-single valued type-2 based on the Mamdani fuzzy model (model a) and an interval SVD-BP hybrid iterative algorithm of two non-single valued type- 2 based on the Mamdani fuzzy model (model two), for short term wind speed time series forecast. Fuzzy controller will enter the exact values into intervals of type-2 fuzzy sets, the main selected membership function with uncertainty Gauss function variance; before rule set main membership function selection has not sure Gauss mean value; rule consequent selection interval set by a Center; of-sets type reduction device. The two models have four inputs, one output model, and rule base containing 24 rules.

\subsection{Design of the Prediction Model of Type-2 Fuzzy}

Ways of carrying out the interval type-2 fuzzy system parameter identification has two kinds: one is in part dependent on the method, first using the given input one output data on the parameters of a fuzzy system identification algorithm through the parameter learning, and then the parameters of a fuzzy system as the initial value to regulate the interval parameter of type-2 fuzzy systems; the two is all independent method, direct identification of parameters of type-2 fuzzy systems with interval parameters selection, arbitrary initial values. The relative ways and second, the prior approach gives an ideal initial interval type-2 fuzzy system parameter identification value, and the results and a fuzzy system can be used to test the baseline type-2 fuzzy system performance therefore, this paper selects, partially dependent method.

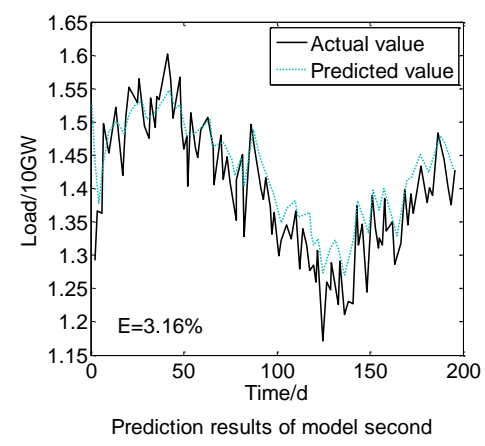

Figure 6. The Actual Value of the Average Relative Error Value of 3.16\%

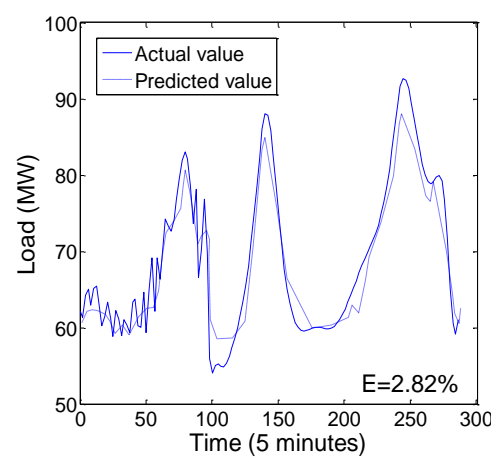

Figure 7. The Actual Value of the Average Relative Error Value of 2.82\% 
With the same number of rules, model two design parameters than the model a more, a total of $2 \times 3+3 \times 3 \times 27+2 \times 27=303$, including input set variance parameter $\mathrm{K}$, before rule set mean parameter $\mathrm{m}_{\mathrm{lk}}$ and the mean variance parameter $\mathrm{L}_{\mathrm{K}}$, rule consequent matter cardiac parameters of $\mathrm{Y}_{\mathrm{L}}$ set, this means that the model two has more adjustable degree of freedom, and thus improve the prediction accuracy, the prediction error is down to $3.16 \%$ (Figure 6); the model three with SVD method in model two is based on rejecting the bad rules, further improve the prediction accuracy, only 12 rules, which makes the prediction error is reduced to $2.82 \%$ (Figure 7 ).

\section{Conclusions}

Compared with the traditional type of fuzzy logic, the processing ability of type- 2 fuzzy logic has more uncertainty, can be directly for uncertainty modeling, the robust control. Signal processing and nonlinear system identification and other fields have broad application prospects, thus also suitable for electric power load forecasting. Accurate power load forecasting is the power system planning, construction, production, scheduling, maintenance and an important basis for the safe operation of the power load fluctuation, and has strong uncertainty, it is difficult to predict, to find a reasonable method to improve the accuracy of power load forecasting is very important, because the ability of type-2 fuzzy logic treatment with excellent uncertainty and become the ideal choice. Power load has strong randomness and difficult to accurately predict problems, is introduced to reduce the fuzzy logic prediction error type-2, built interval type-2 non single valued type-2 fuzzy model is applied to power load time series forecasting, and the use of the SVD SM BP hybrid iterative algorithm to simplify the model rules.

\section{Acknowledgement}

The work was supported by the National Natural Science Foundation Project of China (Grant No.71371011), and Natural Science Foundation of Anhui Province University (Grant No.KJ2013B234), and Humanities and Social Sciences of Anhui Province University (Grant No.SK2015A487), and Hefei University applied mathematics key construction disciplines (NO.2014XK08).

\section{References}

[1] J. M. Mendel, "Type-2 fuzzy sets and systems: an overview", IEEE Computational Intelligence Magazine, vol. 2, no. 2, (2007), pp. 0-29.

[2] L. A. Zadeh, "The concept of a linguistic variable and its application to approximate reasoning-1", Information Sciences, vol. 8, (1975), pp. 199-249.

[3] M. Mizumoto and K. Tanaka, "Fuzzy sets of type-2 under algebraic product and algebraic sum", Fuzzy Sets and systems, vol. 5, (2013), pp. 276-290.

[4] N. N. Karnik, J. M. Mendel and Q. Liang, “Type-2 fuzzy logic systems”, IEEE Transactions on Fuzzy Systems, vol. 7, no. 6, (2013), pp. 642-658.

[5] N. N. Karnik and J. M. Mendel, "Operations on type-2 fuzzy sets", Fuzzy Sets and Systems, vol. 122, (2011), pp. 326-348.

[6] E. Hisdal, "The if then else statement and interval-valued fuzzy sets of higher type", International Journal of Machine Studies, vol. 15, (1981), pp. 384-455.

[7] Q. Liang and J. M. Mendel, "Interval type-2 fuzzy logic systems: theory and design", IEEE Transactions on Fuzzy systems, vol. 8, no. 5, (2012), pp. 534-550.

[8] J. M. Mendel, "On the importance of interval sets in type-2 fuzzy logic systems", Proceedings of Joint $9^{\text {th }}$ IFSA Word Congress and $20^{\text {th }}$ NAFIPS International Conference, Vancouver, BC, Canada, (2011), pp. 1646-1652.

[9] H. Landau, "Prolate spheroidal wave functions, Fourier analysis and uncertainty-III, The dimension of the space of essentially time and band-limited signal", Bell Syst. Tech, (2002). 
[10] K. N. Krishnanand and D. Ghose, “A glowworm swarm optimization based multi-robot system for signal source localization”, Design and Control of Intelligent Robotic Systems, (2009), pp. 53-74.

\section{Authors}

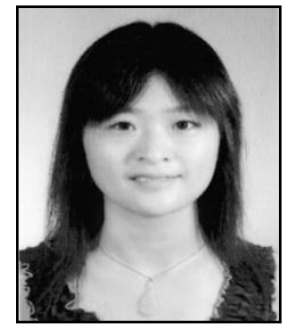

Juan Zhao, she was born in 1980.01.03. She is a woman. She is from BengBu Anhui, China. And she is a professor. 